\title{
A simple proof of Duquesne's theorem on contour processes of conditioned Galton-Watson trees
}

Igor Kortchemski

\begin{abstract}
We give a simple new proof of a theorem of Duquesne, stating that the properly rescaled contour function of a critical aperiodic Galton-Watson tree, whose offspring distribution is in the domain of attraction of a stable law of index $\theta \in(1,2]$, conditioned on having total progeny $n$, converges in the functional sense to the normalized excursion of the continuous-time height function of a strictly stable spectrally positive Lévy process of index $\theta$. To this end, we generalize an idea of Le Gall which consists in using an absolute continuity relation between the conditional probability of having total progeny exactly $n$ and the conditional probability of having total progeny at least $n$. This new method is robust and can be adapted to establish invariance theorems for Galton-Watson trees having $n$ vertices whose degrees are prescribed to belong to a fixed subset of the positive integers.

Keywords. Conditioned Galton-Watson tree, Stable continuous random tree, Scaling limit, Invariance PRINCIPLE.
\end{abstract}

AMS 2000 subject classifications. Primary 60J80,60F17,60G52; secondary 05C05.

\section{Introduction}

In this article, we are interested in the asymptotic behavior of critical Galton-Watson trees whose offspring distribution may have infinite variance. Aldous [1] studied the shape of large critical GaltonWatson trees whose offspring distribution has finite variance and proved that their properly rescaled contour functions converge in distribution in the functional sense to the Brownian excursion. This seminal result has motivated the study of the convergence of other rescaled paths obtained from Galton-Watson trees, such as the Lukasiewicz path (also known as the Harris walk) and the height function. In [21], under an additional exponential moment condition, Marckert \& Mokkadem showed that the rescaled Lukasiewicz path, height function and contour function all converge in distribution to the same Brownian excursion. In parallel, unconditional versions of Aldous' result have been obtained in full generality. More precisely, when the offspring distribution is in the domain of attraction of a stable law of index $\theta \in(1,2]$, Duquesne \& Le Gall [9] showed that the concatenation of rescaled Lukasiewicz paths of a sequence of independent Galton-Watson trees converges in distribution to a strictly stable spectrally positive Lévy process $X$ of index $\theta$, and the concatenation of the associated rescaled height functions (or of the rescaled contour functions) converges in distribution to the so-called continuous-time height function associated with $X$. In the same monograph, Duquesne \& Le Gall explained how to deduce a limit theorem for Galton-Watson trees conditioned on having at

Igor Kortchemski

Université Paris-Sud, Orsay, France e-mail: ifror.kortchemski@normalesup.org 
least $n$ vertices from the unconditional limit theorem. Finally, still in the stable case, Duquesne [8] showed that the rescaled Lukasiewicz path of a Galton-Watson tree conditioned on having $n$ vertices converges in distribution to the normalized excursion of the Lévy process $X$ (thus extending Marckert \& Mokkadem's result) and that the rescaled height and contour functions of a Galton-Watson tree conditioned on having $n$ vertices converge in distribution to the normalized excursion of the continuous-time height function $H^{\text {exc }}$ associated with $X$ (thus extending Aldous' result).

In this work, we give an alternative proof of Duquesne's result, which is based on an idea that appeared in the recent papers [17, 19]. Let us explain our approach after introducing some notation. For every $x \in \mathbb{R}$, let $\lfloor x\rfloor$ denote the greatest integer smaller than or equal to $x$. If $I$ is an interval, let $\mathfrak{C}(I, \mathbb{R})$ be the space of all continuous functions $I \rightarrow \mathbb{R}$ equipped with the topology of uniform convergence on compact subsets of $I$. We also let $\mathbb{D}(I, \mathbb{R})$ be the space of all right-continuous with left limits (càdlàg) functions $I \rightarrow \mathbb{R}$, endowed with the Skorokhod $J_{1}$-topology (see [5, chap. 3], [13, chap. VI] for background concerning the Skorokhod topology). Denote by $\mathbb{P}_{\mu}$ the law of the Galton-Watson tree with offspring distribution $\mu$. The total progeny of a tree $\tau$ will be denoted by $\zeta(\tau)$. Fix $\theta \in(1,2]$ and let $\left(X_{t}\right)_{t \geq 0}$ be the spectrally positive Lévy process with Laplace exponent $\mathbb{E}\left[\exp \left(-\lambda X_{t}\right)\right]=\exp \left(t \lambda^{\theta}\right)$.

(0) We fix a critical offspring distribution $\mu$ in the domain of attraction of a stable law of index $\theta \in(1,2]$. If $U_{1}, U_{2}, \ldots$ are i.i.d. random variables with distribution $\mu$, and $W_{n}=U_{1}+\cdots+U_{n}-n$, there exist positive constants $\left(B_{n}\right)_{n \geq 0}$ such that $W_{n} / B_{n}$ converges in distribution to $X_{1}$.

(i) Fix $a \in(0,1)$. To simplify notation, set $\mathcal{W}^{a,(n)}=\left(\mathcal{W}_{j}^{a,(n)}, 0 \leq j \leq\lfloor n a\rfloor\right)$ where $\mathcal{W}_{j}^{a,(n)}=$ $\mathcal{W}_{j}(\tau) / B_{n}$ and $\mathcal{W}(\tau)$ is the Lukasiewicz path of $\tau$ (see Section 1.2 below for its definition). Then for every function $f_{n}: \mathbb{Z}^{\lfloor a n\rfloor+1} \rightarrow \mathbb{R}_{+}$, the following absolute continuity relation holds:

$$
\mathbb{E}_{\mu}\left[f_{n}\left(\mathcal{W}^{a,(n)}\right) \mid \zeta(\tau)=n\right]=\mathbb{E}_{\mu}\left[f_{n}\left(\mathcal{W}^{a,(n)}\right) D_{n}^{(a)}\left(\mathcal{W}_{\lfloor a n\rfloor}(\tau)\right) \mid \zeta(\tau) \geq n\right]
$$

with a certain function $D_{n}^{(a)}:\{-1,0,1,2, \ldots\} \rightarrow \mathbb{R}_{+}$.

(ii) We establish the existence of a measurable function $\Gamma_{a}: \mathbb{R}_{+} \rightarrow \mathbb{R}_{+}$such that the quantity $\left|D_{n}^{(a)}(j)-\Gamma_{a}\left(j / B_{n}\right)\right|$ goes to 0 as $n \rightarrow \infty$, uniformly in values of $j$ such that $j / B_{n}$ stays in a compact subset of $\mathbb{R}_{+}^{*}$. Furthermore, if $H$ denotes the continuous-time height process associated with $X$ and $\mathbf{N}$ stands for the Itô excursion measure of $X$ above its infimum, we have for every bounded measurable function $F: \mathbb{D}([0, a], \mathbb{R}) \rightarrow \mathbb{R}_{+}$:

$$
\mathbf{N}\left(F\left(\left(H_{t}\right)_{0 \leq t \leq a}\right) \Gamma_{a}\left(X_{a}\right) \mid \zeta>1\right)=\mathbf{N}\left(F\left(\left(H_{t}\right)_{0 \leq t \leq a}\right) \mid \zeta=1\right),
$$

where $\zeta$ is the duration of the excursion under $\mathbf{N}$.

(iii) We show that under $\mathbb{P}_{\mu}[\cdot \mid \zeta(\tau)=n]$, the rescaled height function converges in distribution on $[0, a]$ for every $a \in(0,1)$. To this end, we fix a bounded continuous function $F: \mathbb{D}([0, a], \mathbb{R}) \rightarrow \mathbb{R}_{+}$and apply formula 11 with $f_{n}\left(\mathcal{W}^{a,(n)}\right)=F\left(\frac{B_{n}}{n} H_{\lfloor n t\rfloor}(\tau) ; 0 \leq t \leq a\right)$ where $H(\tau)$ is the height function of the tree $\tau$. Using the previously mentioned result of Duquesne \& Le Gall concerning GaltonWatson trees conditioned on having at least $n$ vertices, we show that we can restrict ourselves to the case where $\mathcal{W}_{\lfloor a n\rfloor}(\tau) / B_{n}$ stays in a compact subset of $\mathbb{R}_{+}^{*}$, so that we can apply (ii) and obtain that:

$$
\begin{aligned}
\lim _{n \rightarrow \infty} \mathbb{E}_{\mu}\left[F\left(\frac{B_{n}}{n} H_{\lfloor n t\rfloor}(\tau) ; 0 \leq t \leq a\right) \mid \zeta(\tau)=n\right] \\
\quad=\lim _{n \rightarrow \infty} \mathbb{E}_{\mu}\left[F\left(\frac{B_{n}}{n} H_{\lfloor n t\rfloor}(\tau) ; 0 \leq t \leq a\right) D_{n}^{(a)}\left(\mathcal{W}_{\lfloor a n\rfloor}(\tau)\right) \mid \zeta(\tau) \geq n\right] \\
=\mathbf{N}\left(F\left(H_{t} ; 0 \leq t \leq a\right) \Gamma_{a}\left(X_{a}\right) \mid \zeta>1\right) \\
=\mathbf{N}\left(F\left(H_{t} ; 0 \leq t \leq a\right) \mid \zeta=1\right) .
\end{aligned}
$$


(iv) By using a relationship between the contour function and the height function which was noticed by Duquesne \& Le Gall in [9], we get that, under $\mathbb{P}_{\mu}[\cdot \mid \zeta(\tau)=n]$, the scaled contour function converges in distribution on $[0, a]$.

(v) By using the time reversal invariance property of the contour function, we deduce that under $\mathbb{P}_{\mu}[\cdot \mid \zeta(\tau)=n]$, the scaled contour function converges in distribution on the whole segment $[0,1]$.

(vi) Using once again the relationship between the contour function and the height function, we deduce that, under $\mathbb{P}_{\mu}[\cdot \mid \zeta(\tau)=n]$, the scaled height function converges in distribution on $[0,1]$.

In the case where the variance of $\mu$ is finite, Le Gall gave an alternative proof of Aldous' theorem in [17. Theorem 6.1] using a similar approach based on a strong local limit theorem. There are additional difficulties in the infinite variance case, since no such theorem is known in this case.

Let us finally discuss the advantage of this new method. Firstly, the proof is simpler and less technical. Secondly, we believe that this approach is robust and can be adapted to other situations. For instance, using the same ideas, we have established invariance theorems for Galton-Watson trees having $n$ vertices whose degrees are prescribed to belong to a fixed subset of the nonnegative integers [15.

The rest of this text is organized as follows. In Section 1, we present the discrete framework by defining Galton-Watson trees and their codings. We explain how the local limit theorem gives information on the asymptotic behavior of large Galton-Watson trees and present the discrete absolute continuity relation appearing in (1). In Section 2, we discuss the continuous framework: we introduce the strictly stable spectrally positive Lévy process, its Itô excursion measure $\mathbf{N}$ and the associated continuous-time height process. We also prove the absolute continuity relation (3). Finally, in Section 3 we give the new proof of Duquesne's theorem by carrying out steps (i-vi).

Acknowledgments. I am deeply indebted to Jean-François Le Gall for insightful discussions and for making many useful suggestions on first versions of this manuscript.

Notation and main assumptions. Throughout this work $\theta \in(1,2]$ is a fixed parameter. We consider a probability distribution $(\mu(j))_{j \geq 0}$ on the nonnegative integers satisfying the following three conditions:

(i) $\mu$ is critical, meaning that $\sum_{k=0}^{\infty} k \mu(k)=1$.

(ii) $\mu$ is in the domain of attraction of a stable law of index $\theta \in(1,2]$. This means that either the variance of $\mu$ is positive and finite, or $\mu([j, \infty))=j^{-\theta} L(j)$, where $L: \mathbb{R}_{+} \rightarrow \mathbb{R}_{+}$is a function such that $L(x)>0$ for $x$ large enough and $\lim _{x \rightarrow \infty} L(t x) / L(x)=1$ for all $t>0$ (such a function is called slowly varying). We refer to [6] or [10, chapter 3.7] for details.

(iii) $\mu$ is aperiodic, which means that the additive subgroup of the integers $\mathbb{Z}$ spanned by $\{j ; \mu(j) \neq 0\}$ is not a proper subgroup of $\mathbb{Z}$.

We introduce condition (iii) to avoid unnecessary complications, but our results can be extended to the periodic case.

In what follows, $\left(X_{t}\right)_{t>0}$ will stand for the spectrally positive Lévy process with Laplace exponent $\mathbb{E}\left[\exp \left(-\lambda X_{t}\right)\right]=\exp \left(t \lambda^{\theta}\right)$ where $t, \lambda \geq 0$ and $p_{1}$ will denote the density of $X_{1}$. Finally, $\nu$ will stand for the probability measure on $\mathbb{Z}$ defined by $\nu(k)=\mu(k+1)$ for $k \geq-1$. Note that $\nu$ has zero mean.

\section{The discrete setting : Galton-Watson trees}

\subsection{Galton-Watson trees}

Definition 1. Let $\mathbb{N}=\{0,1, \ldots\}$ be the set of all nonnegative integers and $\mathbb{N}^{*}=\{1, \ldots\}$. Let also $U$ be the set of all labels: 


$$
U=\bigcup_{n=0}^{\infty}\left(\mathbb{N}^{*}\right)^{n},
$$

where by convention $\left(\mathbb{N}^{*}\right)^{0}=\{\emptyset\}$. An element of $U$ is a sequence $u=u_{1} \cdots u_{j}$ of positive integers, and we set $|u|=j$, which represents the "generation" of $u$. If $u=u_{1} \cdots u_{j}$ and $v=v_{1} \cdots v_{k}$ belong to $U$, we write $u v=u_{1} \cdots u_{j} v_{1} \cdots v_{k}$ for the concatenation of $u$ and $v$. In particular, note that $u \emptyset=\emptyset u=u$. Finally, a rooted ordered tree $\tau$ is a finite subset of $U$ such that:

1. $\emptyset \in \tau$,

2. if $v \in \tau$ and $v=u j$ for some $j \in \mathbb{N}^{*}$, then $u \in \tau$,

3. for every $u \in \tau$, there exists an integer $k_{u}(\tau) \geq 0$ such that, for every $j \in \mathbb{N}^{*}, u j \in \tau$ if and only if $1 \leq j \leq k_{u}(\tau)$.

In the following, by tree we will mean rooted ordered tree. The set of all trees is denoted by $\mathbb{T}$. We will often view each vertex of a tree $\tau$ as an individual of a population whose $\tau$ is the genealogical tree. The total progeny of $\tau$ will be denoted by $\zeta(\tau)=\operatorname{Card}(\tau)$. Finally, if $\tau$ is a tree and $u \in \tau$, we set $T_{u} \tau=\{v \in U ; u v \in \tau\}$, which is itself a tree.

Definition 2. Let $\rho$ be a probability measure on $\mathbb{N}$ with mean less than or equal to 1 and such that $\rho(1)<1$. The law of the Galton-Watson tree with offspring distribution $\rho$ is the unique probability measure $\mathbb{P}_{\rho}$ on $\mathbb{T}$ such that:

1. $\mathbb{P}_{\rho}\left[k_{\emptyset}=j\right]=\rho(j)$ for $j \geq 0$,

2. for every $j \geq 1$ with $\rho(j)>0$, conditionally on $\left\{k_{\emptyset}=j\right\}$, the shifted trees $T_{1} \tau, \ldots, T_{j} \tau$ are i.i.d. with distribution $\mathbb{P}_{\rho}$.

A random tree whose distribution is $\mathbb{P}_{\rho}$ will be called a $\mathrm{GW}_{\rho}$ tree.

\subsection{Coding Galton-Watson trees}

We now explain how trees can be coded by three different functions. These codings are crucial in the understanding of large Galton-Watson trees.

Definition 3. We write $u<v$ for the lexicographical order on the labels $U$ (for example $\emptyset<1<$ $21<22$ ). Consider a tree $\tau$ and order the individuals of $\tau$ in lexicographical order: $\emptyset=u(0)<u(1)<$ $\cdots<u(\zeta(\tau)-1)$. The height process $H(\tau)=\left(H_{n}(\tau), 0 \leq n<\zeta(\tau)\right)$ is defined, for $0 \leq n<\zeta(\tau)$, by $H_{n}(\tau)=|u(n)|$. For technical reasons, we set $H_{k}(\tau)=0$ for $k \geq \zeta(\tau)$. We extend $H(\tau)$ to $\mathbb{R}_{+}$ by linear interpolation by setting $H_{t}(\tau)=(1-\{t\}) H_{\lfloor t\rfloor}(\tau)+\{t\} H_{\lfloor t\rfloor+1}(\tau)$ for $0 \leq t \leq \zeta(\tau)$, where $\{t\}=t-\lfloor t\rfloor$.

Consider a particle that starts from the root and visits continuously all edges at unit speed (assuming that every edge has unit length), going backwards as little as possible and respecting the lexicographical order of vertices. For $0 \leq t \leq 2(\zeta(\tau)-1), C_{t}(\tau)$ is defined as the distance to the root of the position of the particle at time $t$. For technical reasons, we set $C_{t}(\tau)=0$ for $t \in[2(\zeta(\tau)-1), 2 \zeta(\tau)]$. The function $C(\tau)$ is called the contour function of the tree $\tau$. See Figure 1 for an example, and [8, Section 2] for a rigorous definition.

Finally, the Lukasiewicz path $\mathcal{W}(\tau)=\left(\mathcal{W}_{n}(\tau), n \geq 0\right)$ of a tree $\tau$ is defined by $\mathcal{W}_{0}(\tau)=0$, $\mathcal{W}_{n+1}(\tau)=\mathcal{W}_{n}(\tau)+k_{u(n)}(\tau)-1$ for $0 \leq n \leq \zeta(\tau)-1$ and $\mathcal{W}_{k}(\tau)=0$ for $k>\zeta(\tau)$. For $u \geq 0$, we set $\mathcal{W}_{u}(\tau)=\mathcal{W}_{\lfloor u\rfloor}(\tau)$

Note that necessarily $\mathcal{W}_{\zeta(\tau)}(\tau)=-1$.

Let $\left(W_{n} ; n \geq 0\right)$ be a random walk which starts at 0 with jump distribution $\nu(k)=\mu(k+1)$ for $k \geq-1$. For $j \geq 1$, define $\zeta_{j}=\inf \left\{n \geq 0 ; W_{n}=-j\right\}$. 

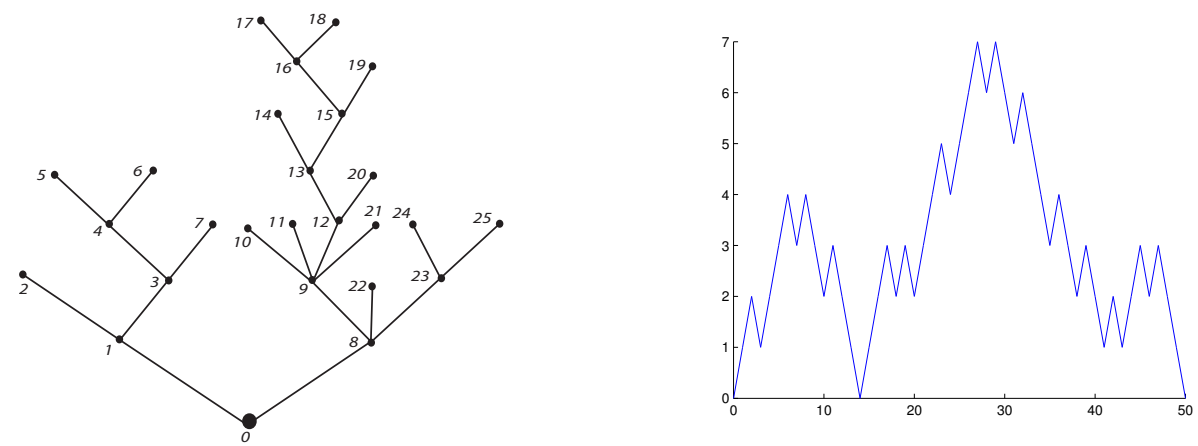

Fig. 1 A tree $\tau$ with its vertices indexed in lexicographical order and its contour function $\left(C_{u}(\tau) ; 0 \leq u \leq 2(\zeta(\tau)-1)\right.$. Here, $\zeta(\tau)=26$.
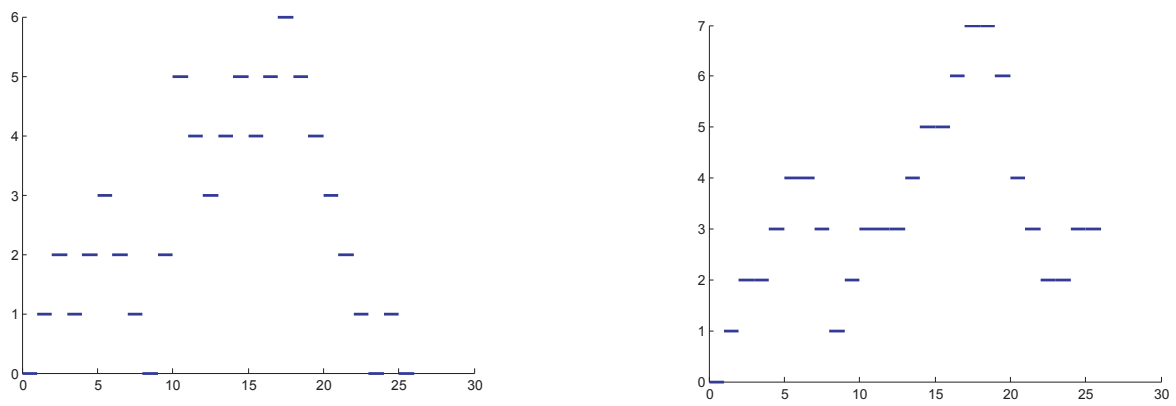

Fig. 2 The Lukasiewicz path $\left(\mathcal{W}_{\lfloor u\rfloor}(\tau) ; 0 \leq u<\zeta(\tau)+1\right)$ and the height function $\left(H_{u}(\tau), 0 \leq u \leq \zeta(\tau)\right)$ of the tree $\tau$ of Fig. 1

Proposition 1. $\left(W_{0}, W_{1}, \ldots, W_{\zeta_{1}}\right)$ has the same distribution as the Lukasiewicz path of a $\mathrm{GW}_{\mu}$ tree. In particular, the total progeny of a $\mathrm{GW}_{\mu}$ tree has the same law as $\zeta_{1}$.

Proof. See [18, Proposition 1.5].

We will also use the following well-known fact (see e.g. Lemma 6.1 in [22] and the discussion that follows).

Proposition 2. For every integers $1 \leq j \leq n$, we have $\mathbb{P}\left[\zeta_{j}=n\right]=\frac{j}{n} \mathbb{P}\left[W_{n}=-j\right]$.

\subsection{Slowly varying functions}

Slowly varying functions appear in the study of domains of attractions of stable laws. Here we recall some properties of these functions in view of future use.

Recall that a positive measurable function $L: \mathbb{R}_{+} \rightarrow \mathbb{R}_{+}$is said to be slowly varying if $L(x)>0$ for $x$ large enough and, for all $t>0, L(t x) / L(x) \rightarrow 1$ as $x \rightarrow \infty$. A useful result concerning these functions is the so-called Representation Theorem, which states that a function $L: \mathbb{R}_{+} \rightarrow \mathbb{R}_{+}$is slowly varying if and only if it can be written in the form:

$$
L(x)=c(x) \exp \left(\int_{1}^{x} \frac{\epsilon(u)}{u} d u\right), \quad x \geq 0,
$$

where $c$ is a nonnegative measurable function having a finite positive limit at infinity and $\epsilon$ is a measurable function tending to 0 at infinity. See e.g. [6, Theorem 1.3.1] for a proof. The following result is then an easy consequence. 
Proposition 3. Fix $\epsilon>0$ and let $L: \mathbb{R}_{+} \rightarrow \mathbb{R}$ be a slowly varying function. There exist two constants $C>1$ and $N>0$ such that $\frac{1}{C} x^{-\epsilon} \leq L(n x) / L(n) \leq C x^{\epsilon}$ for every integer $n \geq N$ and $x \geq 1$.

\subsection{The Local Limit Theorem}

Definition 4. A subset $A \subset \mathbb{Z}$ is said to be lattice if there exist $b \in \mathbb{Z}$ and an integer $d \geq 2$ such that $A \subset b+d \mathbb{Z}$. The largest $d$ for which this statement holds is called the span of $A$. A measure on $\mathbb{Z}$ is said to be lattice if its support is lattice, and a random variable is said to be lattice if its law is lattice.

Remark 1. Since $\mu$ is supposed to be critical and aperiodic, using the fact that $\mu(0)>0$, it is an exercise to check that the probability measure $\nu$ is non-lattice.

Recall that $p_{1}$ is the density of $X_{1}$. It is well known that $p_{1}(0)>0$, that $p_{1}$ is positive, bounded and continuous, and that the absolute value of the derivative of $p_{1}$ is bounded over $\mathbb{R}$ (see e.g. [24, I. 4.]). The following theorem will allow us to find estimates for the probabilities appearing in Proposition 2.

Theorem 1 (Local Limit Theorem). Let $\left(Y_{n}\right)_{n \geq 0}$ be a random walk on $\mathbb{Z}$ started from 0 such that its jump distribution is in the domain of attraction of a stable law of index $\theta \in(1,2]$. Assume that $Y_{1}$ is non-lattice, that $\mathbb{E}\left[Y_{1}\right]=0$ and that $Y_{1}$ takes values in $\mathbb{N} \cup\{-1\}$.

(i) There exists an increasing sequence of positive real numbers $\left(a_{n}\right)_{n \geq 1}$ such that $Y_{n} / a_{n}$ converges in distribution to $X_{1}$.

(ii) We have $\lim _{n \rightarrow \infty} \sup _{k \in \mathbb{Z}}\left|a_{n} \mathbb{P}\left[Y_{n}=k\right]-p_{1}\left(\frac{k}{a_{n}}\right)\right|=0$.

(iii) There exists a slowly varying function $l: \mathbb{R}_{+} \rightarrow \mathbb{R}_{+}$such that $a_{n}=n^{1 / \theta} l(n)$.

Proof. For (i), see [11, Section XVII.5, Theorem 3] and [6, Section 8.4]. The fact that $\left(a_{n}\right)$ may be chosen to be increasing follows from [10, Formula 3.7.2]. For (ii), see [12, Theorem 4.2.1]. For (iii), it is shown in [12, p. 46] that $a_{k n} / a_{n}$ converges to $k^{1 / \theta}$ for every integer $k \geq 1$. Since $\left(a_{n}\right)$ is increasing, a theorem of de Haan (see [6, Theorem 1.10.7]) implies that there exists a slowly varying function $l: \mathbb{R}_{+} \rightarrow \mathbb{R}_{+}$such that $a_{n}=l(n) n^{1 / \theta}$ for every positive integer $n$.

Let $\left(W_{n}\right)_{n \geq 0}$ be as in Proposition 1 a random walk started from 0 with jump distribution $\nu$. Since $\mu$ is in the domain of attraction of a stable law of index $\theta$, it follows that $\nu$ is in the same domain of attraction, and $W_{1}$ is not lattice by Remark 1. Since $\nu$ has zero mean, by the preceding theorem there exists an increasing sequence of positive integers $\left(B_{n}\right)_{n \geq 1}$ such that $B_{n} \rightarrow \infty$ and $W_{n} / B_{n}$ converges in distribution towards $X_{1}$ as $n \rightarrow \infty$. In what follows, the sequence $\left(B_{n}\right)_{n \geq 1}$ will be fixed, and $h: \mathbb{R}_{+} \rightarrow \mathbb{R}_{+}$will stand for a slowly varying function such that $B_{n}=h(n) n^{1 / \theta}$.

Lemma 1. We have:

$$
\text { (i) } \mathbb{P}_{\mu}[\zeta(\tau)=n] \underset{n \rightarrow \infty}{\sim} \frac{p_{1}(0)}{n^{1 / \theta+1} h(n)}, \quad \text { (ii) } \mathbb{P}_{\mu}[\zeta(\tau) \geq n] \underset{n \rightarrow \infty}{\sim} \frac{\theta p_{1}(0)}{n^{1 / \theta} h(n)}
$$

where we write $a_{n} \sim b_{n}$ if $a_{n} / b_{n} \rightarrow 1$.

Proof. We keep the notation of Proposition 1. Proposition 2 gives that:

$$
\mathbb{P}_{\mu}[\zeta(\tau)=n]=\frac{1}{n} \mathbb{P}\left[W_{n}=-1\right] .
$$

For (i), it suffices to notice that the local limit theorem (Theorem 1) and the continuity of $p_{1}$ entail $\mathbb{P}\left[W_{n}=-1\right] \sim p_{1}(0) /\left(h(n) n^{1 / \theta}\right)$. For (ii), we use (i) to write: 


$$
\mathbb{P}_{\mu}[\zeta(\tau) \geq n]=\sum_{k=n}^{\infty}\left(\frac{1}{h(k) k^{1+1 / \theta}} p_{1}(0)+\frac{1}{h(k) k^{1+1 / \theta}} \delta(k)\right)
$$

where $\delta(k)$ tends to 0 as $k \rightarrow \infty$. We can rewrite this in the form:

$$
h(n) n^{1 / \theta} \mathbb{P}_{\mu}[\zeta(\tau) \geq n]=\int_{1}^{\infty} d u f_{n}(u),
$$

where:

$$
f_{n}(u)=\frac{h(n) n^{1 / \theta+1}}{h(\lfloor n u\rfloor)\lfloor n u\rfloor^{1+1 / \theta}}\left(p_{1}(0)+\delta(\lfloor n u\rfloor)\right) .
$$

For fixed $u \geq 1, f_{n}(u)$ tends to $\frac{p_{1}(0)}{u^{1 / \theta+1}}$ as $n \rightarrow \infty$. Choose $\epsilon \in(0,1 / \theta)$. By Proposition 3. for every sufficiently large positive integer $n$ we have $f_{n}(u) \leq C / u^{1+1 / \theta-\epsilon}$ for every $u \geq 1$, where $C$ is a positive constant. The dominated convergence theorem allows us to infer that:

$$
\lim _{n \rightarrow \infty} \int_{1}^{\infty} d u f_{n}(u)=\int_{1}^{\infty} d u \frac{p_{1}(0)}{u^{1 / \theta+1}}=\theta p_{1}(0)
$$

and the desired result follows from (4).

\subsection{Discrete absolute continuity}

The next lemma in another important ingredient of our approach.

Lemma 2 (Le Gall \& Miermont). Fix $a \in(0,1)$. Then, with the notation of Proposition 2, for every $n \geq 1$ and for every bounded nonnegative function $f_{n}$ on $\mathbb{Z}^{\lfloor a n\rfloor+1}$ :

$$
\mathbb{E}\left[f_{n}\left(W_{0}, \ldots, W_{\lfloor n a\rfloor}\right) \mid \zeta_{1}=n\right]=\mathbb{E}\left[f_{n}\left(W_{0}, \ldots, W_{\lfloor n a\rfloor}\right) \frac{\phi_{n-[a n]}\left(W_{\lfloor a n\rfloor}+1\right) / \phi_{n}(1)}{\phi_{n-\lfloor a n\rfloor}^{*}\left(W_{\lfloor a n\rfloor}+1\right) / \phi_{n}^{*}(1)} \mid \zeta_{1} \geq n\right],
$$

where $\phi_{p}(j)=\mathbb{P}\left[\zeta_{j}=p\right]$ and $\phi_{p}^{*}(j)=\mathbb{P}\left[\zeta_{j} \geq p\right]$ for every integers $j \geq 1$ and $p \geq 1$.

Proof. This result follows from an application of the Markov property to the random walk $W$ at time $\lfloor a n\rfloor$. See [19, Lemma 10] for details in a slightly different setting.

\section{The continuous setting: stable Lévy processes}

\subsection{The normalized excursion of the Lévy process and the continuous-time height process}

We follow the presentation of [8]. The underlying probability space will be denoted by $(\Omega, \mathcal{F}, \mathbb{P})$. Recall that $X$ is a strictly stable spectrally positive Lévy process with index $\theta \in(1,2]$ such that for $\lambda>0$ :

$$
\mathbb{E}\left[\exp \left(-\lambda X_{t}\right)\right]=\exp \left(t \lambda^{\theta}\right) .
$$

We denote the canonical filtration generated by $X$ and augmented with the $\mathbb{P}$-negligible sets by $\left(\mathcal{F}_{t}\right)_{t \geq 0}$. See [2] for the proofs of the general assertions of this subsection concerning Lévy processes. In particular, for $\theta=2$ the process $X$ is $\sqrt{2}$ times the standard Brownian motion on the line. Recall that $X$ has the following scaling property: for $c>0$, the process $\left(c^{-1 / \theta} X_{c t}, t \geq 0\right)$ has the same law as $X$. In particular, the density $p_{t}$ of the law of $X_{t}$ enjoys the following scaling property: 


$$
p_{t}(x)=t^{-1 / \theta} p_{1}\left(x t^{-1 / \theta}\right)
$$

for $x \in \mathbb{R}, t>0$. The following notation will be useful: for $s<t$, we set $I_{t}^{s}=\inf _{[s, t]} X$ and $I_{t}=\inf _{[0, t]} X$. Notice that the process $I$ is continuous since $X$ has no negative jumps.

The process $X-I$ is a strong Markov process and 0 is regular for itself with respect to $X-I$. We may and will choose $-I$ as the local time of $X-I$ at level 0 . Let $\left(g_{i}, d_{i}\right), i \in \mathcal{J}$ be the excursion intervals of $X-I$ above 0. For every $i \in \mathcal{J}$ and $s \geq 0$, set $\omega_{s}^{i}=X_{\left(g_{i}+s\right) \wedge d_{i}}-X_{g_{i}}$. We view $\omega^{i}$ as an element of the excursion space $\mathcal{E}$, which is defined by:

$$
\mathcal{E}=\left\{\omega \in \mathbb{D}\left(\mathbb{R}_{+}, \mathbb{R}\right) ; \omega(0)=0 \text { and } \zeta(\omega):=\sup \{s>0 ; \omega(s)>0\} \in(0, \infty)\right\} .
$$

From Itô's excursion theory, the point measure

$$
\mathcal{N}(d t d \omega)=\sum_{i \in \mathcal{I}} \delta_{\left(-I_{g_{i}}, \omega^{i}\right)}
$$

is a Poisson measure on $\mathbb{R}_{+} \times \mathcal{E}$ with intensity $d t \mathbf{N}(d \omega)$, where $\mathbf{N}(d \omega)$ is a $\sigma$-finite measure on $\mathcal{E}$. By classical results, $\mathbf{N}(\zeta>t)=\Gamma(1-1 / \theta)^{-1} t^{-1 / \theta}$. Without risk of confusion, we will also use the notation $X$ for the canonical process on the space $\mathbb{D}\left(\mathbb{R}_{+}, \mathbb{R}\right)$.

We now define the normalized excursion of $X$. Let us first recall the Itô description of the excursion measure (see [7] or [2, Chapter VIII.4] for details). Define for $\lambda>0$ the re-scaling operator $S^{(\lambda)}$ on $\mathcal{E}$ by $S^{(\lambda)}(\omega)=\left(\lambda^{1 / \theta} \omega(s / \lambda), s \geq 0\right)$. Then there exists a unique collection of probability measures $\left(\mathbf{N}_{(a)}, a>0\right)$ on $\mathcal{E}$ such that the following properties hold.

(i) For every $a>0, \mathbf{N}_{(a)}(\zeta=a)=1$.

(ii) For every $\lambda>0$ and $a>0$, we have $S^{(\lambda)}\left(\mathbf{N}_{(a)}\right)=\mathbf{N}_{(\lambda a)}$.

(iii) For every measurable subset $A$ of $\mathcal{E}: \mathbf{N}(A)=\int_{0}^{\infty} \mathbf{N}_{(a)}(A) \frac{d a}{\theta \Gamma(1-1 / \theta) a^{1 / \theta+1}}$.

The probability distribution $\mathbf{N}_{(1)}$ on càdlàg paths with unit lifetime is called the law of the normalized excursion of $X$ and will sometimes be denoted by $\mathbf{N}(\cdot \mid \zeta=1)$. In particular, for $\theta=2, \mathbf{N}_{(1)}$ is the law of $\sqrt{2}$ times the normalized excursion of linear Brownian motion. Informally, $\mathbf{N}(\cdot \mid \zeta=1)$ is the law of an excursion conditioned to have unit lifetime.

We will also use the so-called continuous-time height process $H$ associated with $X$ which was introduced in [20]. If $\theta=2, H$ is set to be equal to $X-I$. If $\theta \in(1,2)$, the process $H$ is defined for every $t \geq 0$ by:

$$
H_{t}:=\lim _{\epsilon \rightarrow 0} \frac{1}{\epsilon} \int_{0}^{t} \mathbb{1}_{\left\{X_{s}<I_{t}^{s}+\epsilon\right\}} d s,
$$

where the limit exists in $\mathbb{P}$-probability and in $\mathbf{N}$-measure on $\{t<\zeta\}$. The definition of $H$ thus makes sense under $\mathbb{P}$ or under $\mathbf{N}$. The process $H$ has a continuous modification both under $\mathbb{P}$ and under $\mathbf{N}$ (see [9, Chapter 1] for details), and from now on we consider only this modification. Using simple scale arguments one can also define $H$ as a continuous random process under $\mathbf{N}(\cdot \mid \zeta=1)$. For our purposes, we will need the fact that, for every $a \geq 0,\left(H_{t}\right)_{0 \leq t \leq a}$ is a measurable function of $\left(X_{t}\right)_{0 \leq t \leq a}$.

\subsection{Absolute continuity property of the Itô measure}

We now present the continuous counterpart of the discrete absolute continuity property appearing in Lemma 2. We follow the presentation of [17] but generalize it to the stable case. The following proposition is classical (see e.g. the proof of Theorem 4.1 in [23, Chapter XII], which establishes the result for Brownian motion). 
Proposition 4. Fix $t>0$. Under the conditional probability measure $\mathbf{N}(\cdot \mid \zeta>t)$, the process $\left(X_{t+s}\right)_{s \geq 0}$ is Markovian with the transition kernels of a strictly stable spectrally positive Lévy process of index $\theta$ stopped upon hitting 0 .

We will also use the following result (see [4, Corollary 2.3] for a proof).

Proposition 5. Set $q_{s}(x)=\frac{x}{s} p_{s}(-x)$ for $x, s>0$. For $x \geq 0$, let $T(x)=\inf \left\{t \geq 0 ; X_{t}<-x\right\}$ be the first passage time of $-X$ above $x$. Then $\mathbb{P}[T(x) \in d t]=q_{t}(x) d t$ for every $x>0$.

Note that $q_{s}$ is a positive continuous function on $(0, \infty)$, for every $s>0$. It is also known that $q_{s}$ is bounded by a constant which is uniform when $s$ varies over $[\epsilon, \infty), \epsilon>0$ (this follows from e.g. [24, I. 4.]).

Proposition 6. For every $a \in(0,1)$ and $x>0$ define:

$$
\Gamma_{a}(x)=\frac{\theta q_{1-a}(x)}{\int_{1-a}^{\infty} d s q_{s}(x)} .
$$

Then for every measurable bounded function $G: \mathbb{D}\left([0, a], \mathbb{R}^{2}\right) \rightarrow \mathbb{R}_{+}$:

$$
\mathbf{N}\left(G\left(\left(X_{t}\right)_{0 \leq t \leq a},\left(H_{t}\right)_{0 \leq t \leq a}\right) \Gamma_{a}\left(X_{a}\right) \mid \zeta>1\right)=\mathbf{N}\left(G\left(\left(X_{t}\right)_{0 \leq t \leq a},\left(H_{t}\right)_{0 \leq t \leq a}\right) \mid \zeta=1\right) .
$$

Proof. Since $\left(H_{t}\right)_{0 \leq t \leq a}$ is a measurable function of $\left(X_{t}\right)_{0 \leq t \leq a}$, it is sufficient to prove that for every bounded measurable function $F: \mathbb{D}([0, a], \mathbb{R}) \rightarrow \mathbb{R}_{+}$:

$$
\mathbf{N}\left(F\left(\left(X_{t}\right)_{0 \leq t \leq a}\right) \Gamma_{a}\left(X_{a}\right) \mid \zeta>1\right)=\mathbf{N}\left(F\left(\left(X_{t}\right)_{0 \leq t \leq a}\right) \mid \zeta=1\right) .
$$

To this end, fix $r \in[0, a]$, let $f, g: \mathbb{R}_{+} \rightarrow \mathbb{R}_{+}$be two bounded continuous functions and let $h: \mathbb{R}_{+}^{*} \rightarrow$ $\mathbb{R}_{+}$be a continuous function. Using the notation of Proposition 5 , we have:

$$
\begin{aligned}
\mathbf{N}\left(f\left(X_{r}\right) h\left(X_{a}\right) g(\zeta) \mathbb{1}_{\{\zeta>a\}}\right) & =\mathbf{N}\left(f\left(X_{r}\right) \mathbb{1}_{\{\zeta>a\}} \mathbb{E}[h(x) g(a+T(x))]_{x=X_{a}}\right) \\
& =\int_{0}^{\infty} d s g(a+s) \mathbf{N}\left(f\left(X_{r}\right) h\left(X_{a}\right) q_{s}\left(X_{a}\right) \mathbb{1}_{\{\zeta>a\}}\right) \\
& =\int_{a}^{\infty} d u g(u) \mathbf{N}\left(f\left(X_{r}\right) h\left(X_{a}\right) q_{u-a}\left(X_{a}\right) \mathbb{1}_{\{\zeta>a\}}\right),
\end{aligned}
$$

where we have used Proposition 4 in the first equality and Proposition 5 in the second equality. Moreover, by property (iii) in subsection 2.1.

$$
\mathbf{N}\left(f\left(X_{r}\right) g(\zeta) \mathbb{1}_{\{\zeta>a\}}\right)=\int_{a}^{\infty} d u \frac{g(u)}{\theta \Gamma(1-1 / \theta) u^{1 / \theta+1}} \cdot \mathbf{N}_{(u)}\left(f\left(X_{r}\right)\right) .
$$

Now observe that (8) (with $h=1$ ) and (9) hold for any bounded continuous function $g$. Since both functions $u \mapsto \mathbf{N}\left(f\left(X_{r}\right) q_{u-a}\left(X_{a}\right) \mathbb{1}_{\{\zeta>a\}}\right)$ and $u \mapsto \mathbf{N}_{(u)}\left(f\left(X_{r}\right)\right)$ are easily seen to be continuous over $(a, \infty)$, it follows that for every $u>a$ :

$$
\mathbf{N}\left(f\left(X_{r}\right) q_{u-a}\left(X_{a}\right) \mathbb{1}_{\{\zeta>a\}}\right)=\frac{1}{\theta \Gamma(1-1 / \theta) u^{1 / \theta+1}} \mathbf{N}_{(u)}\left(f\left(X_{r}\right)\right) .
$$

In particular, for $u=1$ we get:

$$
\mathbf{N}\left(f\left(X_{r}\right) q_{1-a}\left(X_{a}\right) \mathbb{1}_{\{\zeta>a\}}\right)=\frac{1}{\theta \Gamma(1-1 / \theta)} \mathbf{N}_{(1)}\left(f\left(X_{r}\right)\right) .
$$
get:

On the other hand, applying (8) with $g(x)=\mathbb{1}_{\{x>1\}}$ and noting that $\mathbf{N}(\zeta>1)=\frac{1}{\Gamma(1-1 / \theta)}$, we 


$$
\mathbf{N}\left(f\left(X_{r}\right) h\left(X_{a}\right) \mid \zeta>1\right)=\Gamma(1-1 / \theta) \mathbf{N}\left(f\left(X_{r}\right) h\left(X_{a}\right) \mathbb{1}_{\{\zeta>a\}} \int_{1-a}^{\infty} d s q_{s}\left(X_{a}\right)\right) .
$$

By combining $[11$ and $[10$ we conclude that:

$$
\mathbf{N}\left(f\left(X_{r}\right) \frac{\theta q_{1-a}\left(X_{a}\right)}{\int_{1-a}^{\infty} d s q_{s}\left(X_{a}\right)} \mid \zeta>1\right)=\mathbf{N}_{(1)}\left(f\left(X_{r}\right)\right)
$$

One similarly shows that for $0 \leq r_{1}<\cdots<r_{n} \leq a$ and $f_{1}, \ldots, f_{n}: \mathbb{R}_{+} \rightarrow \mathbb{R}_{+}$continuous bounded functions:

$$
\mathbf{N}\left(f_{1}\left(X_{r_{1}}\right) \cdots f_{n}\left(X_{r_{n}}\right) \frac{\theta q_{1-a}\left(X_{a}\right)}{\int_{1-a}^{\infty} d s q_{s}\left(X_{a}\right)} \mid \zeta>1\right)=\mathbf{N}_{(1)}\left(f_{1}\left(X_{r_{1}}\right) \cdots f_{n}\left(X_{r_{n}}\right)\right)
$$

The desired result follows since the Borel $\sigma$-field of $\mathbb{D}([0, a], \mathbb{R})$ is generated by the coordinate functions $X \mapsto X_{r}$ for $0 \leq r \leq a$ (see e.g. [5, Theorem 12.5 (iii)]).

\section{Convergence to the stable tree}

\subsection{An invariance theorem}

We rely on the following theorem, which is similar in spirit to Donsker's invariance theorem (see the concluding remark of [9, Section 2.6] for a proof).

Theorem 2 (Duquesne \& Le Gall). Let $\mathfrak{t}_{n}$ be a random tree distributed according to $\mathbb{P}_{\mu}[\cdot \mid \zeta(\tau) \geq$ $n]$. We have:

$$
\left(\frac{1}{B_{n}} W_{\lfloor n t\rfloor}\left(\mathfrak{t}_{n}\right), \frac{B_{n}}{n} H_{n t}\left(\mathfrak{t}_{n}\right)\right)_{t \geq 0} \underset{n \rightarrow \infty}{\stackrel{(d)}{\longrightarrow}}\left(X_{t}, H_{t}\right)_{0 \leq t \leq 1} \text { under } \mathbf{N}(\cdot \mid \zeta>1) .
$$

\subsection{Convergence of the scaled contour and height functions}

Recall the notation $\phi_{n}(j)=\mathbb{P}\left[\zeta_{j}=n\right]$ and $\phi_{n}^{*}(j)=\mathbb{P}\left[\zeta_{j} \geq n\right]$.

Lemma 3. Fix $\alpha>0$. We have:

(i) $\lim _{n \rightarrow \infty} \sup _{1 \leq k \leq \alpha B_{n}}\left|n \phi_{n}(k)-q_{1}\left(\frac{k}{B_{n}}\right)\right|=0, \quad$ (ii) $\lim _{n \rightarrow \infty} \sup _{1 \leq k \leq \alpha B_{n}}\left|\phi_{n}^{*}(k)-\int_{1}^{\infty} d s q_{s}\left(\frac{k}{B_{n}}\right)\right|=0$.

This has been proved by Le Gall in [17] when $\mu$ has finite variance. In full generality, the proof is technical and is postponed to Section 3.3.

Lemma 4. Fix $a \in(0,1)$. Let $\mathfrak{t}_{n}$ be a random tree distributed according to $\mathbb{P}_{\mu}[\cdot \mid \zeta(\tau)=n]$. Then the following convergence holds in distribution in the space $\mathcal{C}([0, a], \mathbb{R})$ :

$$
\left(\frac{B_{n}}{n} H_{n t}\left(\mathfrak{t}_{n}\right) ; 0 \leq t \leq a\right) \quad \underset{n \rightarrow \infty}{(d)} \quad\left(H_{t} ; 0 \leq t \leq a\right) \text { under } \mathbf{N}(\cdot \mid \zeta=1)
$$

Proof. Recall the notation $\Gamma_{a}$ introduced in Proposition 6. We start by verifying that, for $\alpha>1$, we have:

$$
\lim _{n \rightarrow \infty}\left(\sup _{\frac{1}{\alpha} B_{n} \leq k \leq \alpha B_{n}}\left|\frac{\phi_{n-\lfloor a n\rfloor}(k+1) / \phi_{n}(1)}{\phi_{n-\lfloor a n\rfloor}^{*}(k+1) / \phi_{n}^{*}(1)}-\Gamma_{a}\left(\frac{k}{B_{n}}\right)\right|\right)=0
$$


To this end, we will use the existence of a constant $\delta>0$ such that, for $n$ sufficiently large,

$$
\inf _{\frac{1}{\alpha} B_{n} \leq k \leq \alpha B_{n}} \int_{1}^{\infty} d s q_{s}\left(\frac{k+1}{B_{n-\lfloor a n\rfloor}}\right)>\delta .
$$

The existence of such $\delta$ follows from the fact that, for every $\beta>1, \inf _{\frac{1}{\beta} \leq x \leq \beta} \int_{1}^{\infty} d s q_{s}(x)>0$. We will also need the fact that for every $\beta>1$ there exists a constant $C>0$ such :

$$
\sup _{\frac{1}{\beta} \leq x \leq \beta} q_{1}(x) \leq C, \quad \sup _{\frac{1}{\beta} \leq x \leq \beta} \int_{1}^{\infty} d s q_{s}(x) \leq C .
$$

This is a consequence of the fact that $q_{1}$ is bounded for the first inequality, and the second inequality follows from the scaling property (7) combined with the fact that $p_{1}$ is bounded (see e.g. [24, I. 4.]). To establish $[12$, we first show that

$$
\lim _{n \rightarrow \infty}\left(\sup _{\frac{1}{\alpha} B_{n} \leq k \leq \alpha B_{n}}\left|\frac{\phi_{n-\lfloor a n\rfloor}(k+1) / \phi_{n}(1)}{\phi_{n-\lfloor a n\rfloor}^{*}(k+1) / \phi_{n}^{*}(1)}-\theta \frac{\frac{1}{1-a} q_{1}\left(\frac{k+1}{B_{n-\lfloor a n\rfloor}}\right)}{\int_{1}^{\infty} d s q_{s}\left(\frac{k+1}{B_{n-\lfloor a n\rfloor}}\right)}\right|\right)=0
$$

Since $B_{n-\lfloor a n\rfloor} / B_{n} \rightarrow(1-a)^{1 / \theta}$ as $n \rightarrow \infty$, Lemma 3 garanties the existence of two sequences $\left(\varepsilon_{k, n}^{(1)}, \varepsilon_{k, n}^{(2)}\right)_{k, n \geq 1}$ such that

$(n-\lfloor a n\rfloor) \phi_{n-\lfloor a n\rfloor}(k+1)=q_{1}\left(\frac{k+1}{B_{n-\lfloor a n\rfloor}}\right)+\varepsilon_{k, n}^{(1)}, \quad \phi_{n-\lfloor a n\rfloor}^{*}(k+1)=\int_{1}^{\infty} d s q_{s}\left(\frac{k+1}{B_{n-\lfloor a n\rfloor}}\right)+\varepsilon_{k, n}^{(2)}$

and such that $\max \left(\varepsilon_{k, n}^{(1)}, \varepsilon_{k, n}^{(2)}\right) \rightarrow 0$ as $n \rightarrow \infty$, uniformly in $1 / \alpha \cdot B_{n} \leq k \leq \alpha B_{n}$. To simplify notation set $m_{n}=n-\lfloor a n\rfloor$. By $(14\rfloor$ and the fact that $B_{m_{n}} / B_{n} \rightarrow(1-a)^{1 / \theta}$, there exists $C>0$ such that for $n$ sufficiently large and $1 / \alpha \cdot B_{n} \leq k \leq \alpha B_{n}$ :

$$
\begin{aligned}
\left|\frac{m_{n} \phi_{m_{n}}(k+1)}{\phi_{m_{n}}^{*}(k+1)}-\frac{q_{1}\left(\frac{k+1}{B_{m_{n}}}\right)}{\int_{1}^{\infty} d s q_{s}\left(\frac{k+1}{B_{m_{n}}}\right)}\right| & =\left|\frac{\varepsilon_{k, n}^{(1)} \cdot \int_{1}^{\infty} d s q_{s}\left(\frac{k+1}{B_{m_{n}}}\right)-\varepsilon_{k, n}^{(2)} \cdot q_{1}\left(\frac{k+1}{B_{m_{n}}}\right)}{\int_{1}^{\infty} d s q_{s}\left(\frac{k+1}{B_{m_{n}}}\right) \cdot\left(\int_{1}^{\infty} d s q_{s}\left(\frac{k+1}{B_{m_{n}}}\right)+\varepsilon_{k, n}^{(2)}\right)}\right| \\
& \leq \frac{2 C}{\delta^{2}} \cdot \sup _{\frac{1}{\alpha} B_{n} \leq k \leq \alpha B_{n}} \max \left(\varepsilon_{k, n}^{(1)}, \varepsilon_{k, n}^{(2)}\right),
\end{aligned}
$$

where we have used 130 for the last inequality. This, combined with the fact that $\phi_{n}^{*}(1) /\left(n \phi_{n}(1)\right) \rightarrow \theta$ as $n \rightarrow \infty$ by Lemma 1] implies (15). Then our claim (12) follows the scaling property (7) and the continuity of $\Gamma_{a}$.

We shall now prove another useful result before introducing some notation. Fix $\alpha>1$. Let $g_{n}: \mathbb{R}^{\lfloor a n\rfloor+1} \rightarrow \mathbb{R}_{+}$be a bounded measurable function. To simplify notation, for $x_{0}, \ldots, x_{\lfloor a n\rfloor} \in \mathbb{R}$, set

$$
G_{n}\left(x_{0}, \ldots, x_{\lfloor a n\rfloor}\right)=g_{n}\left(x_{0}, \ldots, x_{\lfloor a n\rfloor}\right) \mathbb{1}_{x_{\lfloor a n\rfloor} \in\left[\frac{1}{\alpha} B_{n}, \alpha B_{n}\right]}
$$

and, for a tree $\tau$, set

$$
\widetilde{G}_{n}(\tau)=g_{n}\left(\mathcal{W}_{0}(\tau), \mathcal{W}_{1}(\tau), \ldots, \mathcal{W}_{\lfloor a n\rfloor}(\tau)\right) \mathbb{1}_{\left\{\mathcal{W}_{\lfloor n a\rfloor}(\tau) \in\left[\frac{1}{\alpha} B_{n}, \alpha B_{n}\right]\right\}} .
$$

We claim that

$$
\lim _{n \rightarrow \infty}\left|\mathbb{E}\left[\widetilde{G}_{n}\left(\mathfrak{t}_{n}\right)\right]-\mathbb{E}_{\mu}\left[\widetilde{G}_{n}(\tau) \Gamma_{a}\left(\frac{\mathcal{W}_{\lfloor a n\rfloor}(\tau)}{B_{n}}\right) \mid \zeta(\tau) \geq n\right]\right|=0
$$

Indeed, using successively Proposition 1 and (5), we have: 


$$
\begin{aligned}
\mathbb{E}\left[\widetilde{G}_{n}\left(\mathfrak{t}_{n}\right)\right]-\mathbb{E}_{\mu}\left[\widetilde{G}_{n}(\tau) \Gamma_{a}\left(\frac{\mathcal{W}_{\lfloor a n\rfloor}(\tau)}{B_{n}}\right) \mid \zeta(\tau) \geq n\right] \\
\quad=\mathbb{E}\left[G_{n}\left(W_{0}, \ldots, W_{\lfloor n a\rfloor}\right) \mid \zeta_{1}=n\right]-\mathbb{E}\left[G_{n}\left(W_{0}, \ldots, W_{\lfloor n a\rfloor}\right) \Gamma_{a}\left(\frac{W_{\lfloor a n\rfloor}}{B_{n}}\right) \mid \zeta_{1} \geq n\right] \\
\quad=\mathbb{E}\left[G_{n}\left(W_{0}, \ldots, W_{\lfloor n a\rfloor}\right)\left(\frac{\phi_{n-[a n\rfloor}\left(W_{\lfloor a n\rfloor}+1\right) / \phi_{n}(1)}{\phi_{n-\lfloor a n\rfloor}^{*}\left(W_{\lfloor a n\rfloor}+1\right) / \phi_{n}^{*}(1)}-\Gamma_{a}\left(\frac{W_{\lfloor a n\rfloor}}{B_{n}}\right)\right) \mid \zeta_{1} \geq n\right] .
\end{aligned}
$$

Our claim (16) then follows from (12).

We finally return to the proof of Lemma 4. Let $F: \mathbb{D}([0, a], \mathbb{R}) \rightarrow \mathbb{R}_{+}$be a bounded continuous function. We also set $F_{n}(\tau)=F\left(\frac{B_{n}}{n} H_{\lfloor n t\rfloor}(\tau) ; 0 \leq t \leq a\right)$. Since $\left(H_{0}(\tau), H_{1}(\tau), \ldots, H_{\lfloor a n\rfloor}(\tau)\right)$ is a measurable function of $\left(\mathcal{W}_{0}(\tau), \mathcal{W}_{1}(\tau), \ldots, \mathcal{W}_{\lfloor\text {an }}(\tau)\right)$ (see [18, Prop 1.2]), by (16) we get:

$$
\lim _{n \rightarrow \infty}\left|\mathbb{E}\left[F_{n}\left(\mathfrak{t}_{n}\right) \mathbb{1}_{A_{n}^{\alpha}\left(\mathfrak{t}_{n}\right)}\right]-\mathbb{E}_{\mu}\left[F_{n}(\tau) \mathbb{1}_{A_{n}^{\alpha}(\tau)} \Gamma_{a}\left(\frac{\mathcal{W}_{\lfloor\text {an」 }}(\tau)}{B_{n}}\right) \mid \zeta(\tau) \geq n\right]\right|=0 .
$$

By Theorem 2, the law of

$$
\left(\left(\frac{B_{n}}{n} H_{\lfloor n t\rfloor}(\tau) ; 0 \leq t \leq a\right), \frac{1}{B_{n}} \mathcal{W}_{\lfloor a n\rfloor}(\tau)\right)
$$

under $\mathbb{P}_{\mu}[\cdot \mid \zeta(\tau) \geq n]$ converges towards the law of $\left(\left(H_{t} ; 0 \leq t \leq a\right), X_{a}\right)$ under $\mathbf{N}(\cdot \mid \zeta>1)$ (for the convergence of the second component we have also used the fact that $X$ is almost surely continuous at $a$ ). Thus:

$$
\begin{aligned}
\lim _{n \rightarrow \infty} \mathbb{E}\left[F_{n}\left(\mathfrak{t}_{n}\right) \mathbb{1}_{\left.\left\{\mathcal{W}_{\lfloor n a\rfloor}\left(\mathfrak{t}_{n}\right) \in\left[\frac{1}{\alpha} B_{n}, \alpha B_{n}\right]\right\}\right]}\right. & =\mathbf{N}\left(F\left(H_{t} ; 0 \leq t \leq a\right) \Gamma_{a}\left(X_{a}\right) \mathbb{1}_{\left\{X_{a} \in\left[\frac{1}{\alpha}, \alpha\right]\right\}} \mid \zeta>1\right) \\
& =\mathbf{N}\left(F\left(H_{t} ; 0 \leq t \leq a\right) \mathbb{1}_{\left\{X_{a} \in\left[\frac{1}{\alpha}, \alpha\right]\right\}} \mid \zeta=1\right),
\end{aligned}
$$

where we have used Proposition 6 in the second equality.

By taking $F \equiv 1$, we obtain:

$$
\lim _{n \rightarrow \infty} \mathbb{P}\left[\mathcal{W}_{\lfloor n a\rfloor}\left(\mathfrak{t}_{n}\right) \in\left[\frac{1}{\alpha} B_{n}, \alpha B_{n}\right]\right]=\mathbf{N}\left(X_{a} \in\left[\frac{1}{\alpha}, \alpha\right] \mid \zeta=1\right) .
$$

This last quantity tends to 1 as $\alpha \rightarrow \infty$. By choosing $\alpha>1$ sufficiently large, we easily deduce from the convergence (17) that:

$$
\lim _{n \rightarrow \infty} \mathbb{E}\left[F\left(\frac{B_{n}}{n} H_{\lfloor n t\rfloor}\left(\mathfrak{t}_{n}\right) ; 0 \leq t \leq a\right)\right]=\mathbf{N}\left(F\left(H_{t} ; 0 \leq t \leq a\right) \mid \zeta=1\right) .
$$

The path continuity of $H$ under $\mathbf{N}(\cdot \mid \zeta=1)$ then implies the claim of Lemma 4

Theorem 3. Let $\mathfrak{t}_{n}$ be a random tree distributed according to $\mathbb{P}_{\mu}[\cdot \mid \zeta(\tau)=n]$. Then:

$$
\left(\frac{B_{n}}{n} H_{n t}\left(\mathfrak{t}_{n}\right), \frac{B_{n}}{n} C_{2 n t}\left(\mathfrak{t}_{n}\right)\right)_{0 \leq t \leq 1} \quad \underset{n \rightarrow \infty}{\stackrel{(d)}{\longrightarrow}} \quad\left(H_{t}, H_{t}\right)_{0 \leq t \leq 1} \text { under } \mathbf{N}(\cdot \mid \zeta=1) .
$$

Proof. The proof consists in showing that the scaled height process is close to the scaled contour process and then using a time-reversal argument in order to show that the convergence holds on the whole segment $[0,1]$. To this end, we adapt [ 8 , Remark 3.2] and [9, Section 2.4] to our context. For $0 \leq p<n$ set $b_{p}=2 p-H_{p}\left(\mathfrak{t}_{n}\right)$ so that $b_{p}$ represents the time needed by the contour process to reach the $(p+1)$-st individual of $\mathfrak{t}_{n}$ (in the lexicographical order). Also set $b_{n}=2(n-1)$. Note that $C_{b_{p}}=H_{p}$. From this observation, we get:

$$
\sup _{t \in\left[b_{p}, b_{p+1}\right]}\left|C_{t}\left(\mathfrak{t}_{n}\right)-H_{p}\left(\mathfrak{t}_{n}\right)\right| \leq\left|H_{p+1}\left(\mathfrak{t}_{n}\right)-H_{p}\left(\mathfrak{t}_{n}\right)\right|+1 .
$$


for $0 \leq p<n$. Then define the random function $g_{n}:[0,2 n] \rightarrow \mathbb{N}$ by setting $g_{n}(t)=k$ if $t \in\left[b_{k}, b_{k+1}\right)$ and $k<n$, and $g_{n}(t)=n$ if $t \in[2(n-1), 2 n]$ so that for $t<2(n-1), g_{n}(t)$ is the index of the last individual which has been visited by the contour function up to time $t$ if the individuals are indexed $0,1, \ldots, n-1$ in lexicographical order. Finally, set $\widetilde{g}_{n}(t)=g_{n}(n t) / n$. Fix $a \in(0,1)$. Then, by (18):

$$
\sup _{t \leq \leq \frac{\left.b_{a}\right\rfloor a}{n}}\left|\frac{B_{n}}{n} C_{n t}\left(\mathfrak{t}_{n}\right)-\frac{B_{n}}{n} H_{n \widetilde{g}_{n}(t)}\left(\mathfrak{t}_{n}\right)\right| \leq \frac{B_{n}}{n}+\frac{B_{n}}{n} \sup _{k \leq\lfloor a n\rfloor}\left|H_{k+1}\left(\mathfrak{t}_{n}\right)-H_{k}\left(\mathfrak{t}_{n}\right)\right|,
$$

which converges in probability to 0 by Lemma 4 and the path continuity of $\left(H_{t}\right)$. On the other hand it follows from the definition of $b_{n}$ that:

$$
\sup _{\substack{t \leq \frac{b_{\lfloor a n\rfloor}}{n}}}\left|\widetilde{g}_{n}(t)-\frac{t}{2}\right| \leq \frac{1}{2 B_{n}} \sup _{k \leq a n} \frac{B_{n}}{n} H_{k}\left(\mathfrak{t}_{n}\right)+\frac{1}{n} \stackrel{(\mathbb{P})}{\longrightarrow} \quad 0
$$

by Lemma 4 Finally, by the definition of $b_{n}$ and using Lemma 4 we see that $\frac{b_{\lfloor a n\rfloor}}{n}$ converges in probability towards $2 a$ and that $\frac{B_{n}}{n} \sup _{t \leq 2 a}\left|H_{n \widetilde{g}_{n}(t)}\left(\mathfrak{t}_{n}\right)-H_{n t / 2}\left(\mathfrak{t}_{n}\right)\right|$ converges in probability towards 0 as $n \rightarrow \infty$. Using (19), we conclude that:

$$
\frac{B_{n}}{n} \sup _{0 \leq t \leq a}\left|C_{2 n t}\left(\mathfrak{t}_{n}\right)-H_{n t}\left(\mathfrak{t}_{n}\right)\right| \stackrel{(\mathbb{P})}{\longrightarrow} 0 .
$$

Together with Lemma 4, this implies:

$$
\left(\frac{B_{n}}{n} C_{2 n t}\left(\mathfrak{t}_{n}\right) ; 0 \leq t \leq a\right) \quad \stackrel{(d)}{\longrightarrow} \quad\left(H_{t} ; 0 \leq t \leq a\right) \text { under } \mathbf{N}(\cdot \mid \zeta=1) .
$$

Since $\left(C_{t}\left(\mathfrak{t}_{n}\right) ; 0 \leq t \leq 2 n-2\right)$ and $\left(C_{2 n-2-t}\left(\mathfrak{t}_{n}\right) ; 0 \leq t \leq 2 n-2\right)$ have the same distribution, it follows that:

$$
\left(\frac{B_{n}}{n} C_{2 n t}\left(\mathfrak{t}_{n}\right) ; 0 \leq t \leq 1\right) \quad \stackrel{(d)}{\longrightarrow} \quad\left(H_{t} ; 0 \leq t \leq 1\right) \text { under } \mathbf{N}(\cdot \mid \zeta=1) .
$$

See the last paragraph of the proof of Theorem 6.1 in [17] for details.

Finally, we show that this convergence in turn entails the convergence of the rescaled height function of $\mathfrak{t}_{n}$ on the whole segment $[0,1]$. To this end, we verify that convergence 200 remains valid for $a=1$. First note that:

$$
\sup _{0 \leq t \leq 2}\left|\widetilde{g}_{n}(t)-\frac{t}{2}\right| \leq \frac{1}{2 n} \sup _{k \leq n} H_{k}\left(\mathfrak{t}_{n}\right)+\frac{1}{n}=\frac{1}{2 B_{n}} \sup _{k \leq 2 n} \frac{B_{n}}{n} C_{k}\left(\mathfrak{t}_{n}\right)+\frac{1}{n} \stackrel{(\mathbb{P})}{\longrightarrow} \quad 0
$$

by 21. Secondly, it follows from 18 that:

$$
\begin{aligned}
\sup _{0 \leq t \leq 2}\left|\frac{B_{n}}{n} C_{n t}\left(\mathfrak{t}_{n}\right)-\frac{B_{n}}{n} H_{n \widetilde{g}_{n}(t)}\right| & \leq \frac{B_{n}}{n}+\frac{B_{n}}{n} \sup _{k \leq n-1}\left|H_{k+1}\left(\mathfrak{t}_{n}\right)-H_{k}\left(\mathfrak{t}_{n}\right)\right| \\
& =\frac{B_{n}}{n}+\frac{B_{n}}{n} \sup _{k \leq n-1}\left|C_{\frac{b_{k+1}}{n} n}\left(\mathfrak{t}_{n}\right)-C_{\frac{b_{k}}{n} n}\left(\mathfrak{t}_{n}\right)\right| .
\end{aligned}
$$

By (21), in order to prove that the latter quantity tends to 0 in probability, it is sufficient to verify that $\sup _{k \leq n}\left|\frac{b_{k+1}}{n}-\frac{b_{k}}{n}\right|$ converges to 0 in probability. But by the definition of $b_{n}$ :

$$
\sup _{k \leq n}\left|\frac{b_{k+1}}{n}-\frac{b_{k}}{n}\right|=\sup _{k \leq n}\left|\frac{2+H_{k}\left(\mathfrak{t}_{n}\right)-H_{k+1}\left(\mathfrak{t}_{n}\right)}{n}\right| \leq \frac{2}{n}+2 \sup _{k \leq n} \frac{H_{k}\left(\mathfrak{t}_{n}\right)}{n}
$$


which converges in probability to 0 as in 22 . As a consequence:

$$
\frac{B_{n}}{n} \sup _{0 \leq t \leq 1}\left|C_{2 n t}\left(\mathfrak{t}_{n}\right)-H_{n \widetilde{g}_{n}(2 t)}\left(\mathfrak{t}_{n}\right)\right| \stackrel{(\mathbb{P})}{\longrightarrow} 0 .
$$

By 21], we get that:

$$
\left(\frac{B_{n}}{n} H_{n \widetilde{g}_{n}(2 t)}\left(\mathfrak{t}_{n}\right)\right)_{0 \leq t \leq 1} \underset{n \rightarrow \infty}{\stackrel{(d)}{\longrightarrow}}\left(H_{t}\right)_{0 \leq t \leq 1} \text { under } \mathbf{N}(\cdot \mid \zeta=1) .
$$

Combining this with 22 , we conclude that:

$$
\left(\frac{B_{n}}{n} C_{2 n t}\left(\mathfrak{t}_{n}\right), \frac{B_{n}}{n} H_{n t}\left(\mathfrak{t}_{n}\right)\right)_{0 \leq t \leq 1} \underset{n \rightarrow \infty}{\stackrel{(d)}{\longrightarrow}}\left(H_{t}, H_{t}\right)_{0 \leq t \leq 1} \text { under } \mathbf{N}(\cdot \mid \zeta=1) .
$$

This completes the proof.

Remark 2. If we see the tree $t_{n}$ as a finite metric space using its graph distance, this theorem implies that $\mathfrak{t}_{n}$, suitably rescaled, converges in distribution to the $\theta$-stable tree, in the sense of the GromovHausdorff distance on isometry classes of compact metric spaces (see e.g. [18, Section 2] for details).

Remark 3. When the mean value of $\mu$ is greater than one, it is possible to replace $\mu$ with a critical probability distribution belonging to the same exponential family as $\mu$ without changing the distribution of $\mathfrak{t}_{n}$ (see [14]). Consequently, the theorem holds in the supercritical case as well. The case where $\mu$ is subcritical and $\mu(i) \sim L(i) / i^{1+\theta}$ as $i \rightarrow \infty$ has been treated in [16. However, in full generality, the non-critical subcritical case remains open.

\subsection{Proof of the technical lemma}

In this section, we prove Lemma 3.

Proof (of Lemma 3). We first prove (i). By the local limit theorem (Theorem 1 (ii)), we have, for $k \geq 1$ and $j \in \mathbb{Z}$ :

$$
\left|B_{n} \mathbb{P}\left[W_{n}=j\right]-p_{1}\left(\frac{j}{B_{n}}\right)\right| \leq \epsilon(n),
$$

where $\epsilon(n) \rightarrow 0$. By Proposition 2 we have $n \phi_{n}(j)=j \mathbb{P}\left[W_{n}=-j\right]$. Since $\frac{j}{B_{n}} p_{1}\left(-\frac{j}{B_{n}}\right)=q_{1}\left(\frac{j}{B_{n}}\right)$, we have for $1 \leq j \leq \alpha B_{n}$ :

$$
\left|n \phi_{n}(j)-q_{1}\left(\frac{j}{B_{n}}\right)\right|=\frac{j}{B_{n}}\left|B_{n} \mathbb{P}\left[W_{n}=-j\right]-p_{1}\left(\frac{j}{B_{n}}\right)\right| \leq \alpha \epsilon(n) .
$$

This completes the proof of (i).

For (ii), first note that by the definition of $q_{s}$ and the scaling property (7):

$$
\int_{1}^{\infty} d s q_{s}\left(\frac{j}{B_{n}}\right)=\int_{1}^{\infty} \frac{j / B_{n}}{s^{1 / \theta+1}} p_{1}\left(-\frac{j / B_{n}}{s^{1 / \theta}}\right) d s .
$$

By Proposition 2 and the local limit theorem:

$$
\left|\phi_{n}^{*}(j)-\sum_{k=n}^{\infty} \frac{j}{k B_{k}} p_{1}\left(-\frac{j}{B_{k}}\right)\right|=\left|\sum_{k=n}^{\infty}\left(\frac{j}{k} \mathbb{P}\left[W_{k}=-j\right]-\frac{j}{k B_{k}} p_{1}\left(-\frac{j}{B_{k}}\right)\right)\right| \leq \sum_{k=n}^{\infty} \frac{j}{k B_{k}} \epsilon(k),
$$

where $\epsilon(n) \rightarrow 0$. Then write: 


$$
\begin{aligned}
& \left|\sum_{k=n}^{\infty} \frac{j}{k B_{k}} p_{1}\left(-\frac{j}{B_{k}}\right)-\int_{1}^{\infty} d s \frac{j / B_{n}}{s^{1 / \theta+1}} p_{1}\left(-\frac{j / B_{n}}{s^{1 / \theta}}\right)\right| \\
& \quad \leq \int_{1}^{\infty} d s\left|\frac{j n}{B_{\lfloor n s\rfloor}\lfloor n s\rfloor}-\frac{j / B_{n}}{s^{1 / \theta+1}}\right| p_{1}\left(-\frac{j}{B_{\lfloor n s\rfloor}}\right)+\int_{1}^{\infty} d s \frac{j / B_{n}}{s^{1 / \theta+1}}\left|p_{1}\left(-\frac{j}{B_{\lfloor n s\rfloor}}\right)-p_{1}\left(-\frac{j / B_{n}}{s^{1 / \theta}}\right)\right| .
\end{aligned}
$$

Denote the first term of the right-hand side by $P(n, j)$ and the second term by $Q(n, j)$. Since $p_{1}$ is bounded by a constant which we will denote by $M$, we have for $1 \leq j \leq \alpha B_{n}$ :

$$
P(n, j) \leq \alpha M \int_{1}^{\infty} d s \frac{1}{s^{1 / \theta+1}}\left|\frac{n B_{n} s^{1 / \theta+1}}{B_{\lfloor n s\rfloor}\lfloor n s\rfloor}-1\right| .
$$

For fixed $s \geq 1, \frac{1}{s^{1 / \theta+1}}\left|\frac{n B_{n} s^{1 / \theta+1}}{B_{\lfloor n s\rfloor}\lfloor n s\rfloor}-1\right|$ tends to 0 as $n \rightarrow \infty$, and using Proposition 3 , the same quantity is bounded by an integrable function independent of $n$. The dominated convergence theorem thus shows that $P(n, j) \rightarrow 0$ uniformly in $1 \leq j \leq \alpha B_{n}$. Let us now bound $Q(n, j)$ for $1 \leq j \leq \alpha B_{n}$. Since the absolute value of the derivative of $p_{1}$ is bounded by a constant which we will denote by $M^{\prime}$, we have:

$$
Q(n, j) \leq M^{\prime} \int_{1}^{\infty} d s \frac{j / B_{n}}{s^{1 / \theta+1}}\left|\frac{j}{B_{\lfloor n s\rfloor}}-\frac{j / B_{n}}{s^{1 / \theta}}\right| \leq \alpha^{2} M^{\prime} \int_{1}^{\infty} d s \frac{1}{s^{2 / \theta+1}}\left|\frac{B_{n} s^{1 / \theta}}{B_{\lfloor n s\rfloor}}-1\right| .
$$

The right-hand side tends to 0 by the same argument we used for $P(n, j)$. We have thus proved that:

$$
\lim _{n \rightarrow \infty} \sup _{1 \leq j \leq \alpha B_{n}}\left|\sum_{k=n}^{\infty} \frac{j}{k B_{k}} p_{1}\left(-\frac{k}{B_{k}}\right)-\int_{1}^{\infty} d s q_{s}\left(\frac{j}{B_{n}}\right)\right|=0 .
$$

One finally shows that $\sum_{k=n}^{\infty} \frac{j}{k B_{k}} \epsilon(k)$ tends to 0 as $n \rightarrow \infty$ uniformly in $1 \leq j \leq \alpha B_{n}$ by noticing that:

$$
\sup _{n \geq 1} \sup _{1 \leq j \leq \alpha B_{n}}\left(\sum_{k=n}^{\infty} \frac{j}{k B_{k}}\right) \leq \alpha \sup _{n \geq 1}\left(\sum_{k=n}^{\infty} \frac{B_{n}}{k B_{k}}\right)<\infty .
$$

This completes the proof.

\section{References}

1. D. Aldous, The continuum random tree III, Ann. Probab. 21, 248-289 (1993).

2. J. Bertoin, Lévy processes, Cambridge Univ. Press (1996).

3. J. Bennies, G. Kersting, A random walk approach to Galton-Watson trees, J. Theoret. Probab. 13, 777-803, (2000).

4. J. Bertoin, Subordinators, Lévy processes with no negative jumps and branching processes, MaPhySto Lecture Notes Series No. 8 (2000).

5. P. Billingsley, Convergence of probability measures, Second Edition, Wiley Series in Probability and Statistics: Probability and Statistics. John Wiley and Sons, Inc., New York (1999).

6. N.H. Bingham, C.M. Goldie, J.L. Teugels, Regular variation, Encyclopedia of Mathematics and Its Applications, vol. 27, Cambridge University Press, Cambridge, (1987).

7. L. Chaumont, Excursion normalisée, méandre et pont pour les processus de Lévy stables, Bull. Sci. Math. 121(5), 377-403 (1997).

8. T. Duquesne, A limit theorem for the contour process of conditioned Galton-Watson trees, Ann. Probab. 31, 996-1027 (2003).

9. T. Duquesne, J.-F. Le Gall, Random Trees, Lévy Processes and Spatial Branching Processes, Astérisque 281 (2002).

10. R. Durrett, Probability: Theory and Examples, 4th edition, Cambridge U. Press (2010).

11. W. Feller, An Introduction to Probability Theory and Its Applications, Vol. 2, 2nd ed. New York, John Wiley (1971).

12. I.A. Ibragimov, Y.V. Linnik, Independent and Stationary Sequences of Independent Random Variables, WoltersNoordhoff, Groningen (1971). 
13. J. Jacod, A. Shiryaev, Limit Theorems for Stochastic Processes. Series: Grundlehren der mathematischen Wissenschaften, Vol. 288, 2nd ed. (2003).

14. D.P. Kennedy, The Galton-Watson process conditioned on the total progeny, J. Appl. Probab. 12, $800-806$ (1975).

15. I. Kortchemski, Invariance principles for Galton-Watson trees conditioned on the number of leaves, Stoch. Proc. Appl. 122 3126-3172 (2012).

16. I. Kortchemski, Limit theorems for conditioned non-generic Galton-Watson trees, preprint, arXiv1205.3145.

17. J.-F. Le Gall, Itô's excursion theory and random trees, Stochastic Process. Appl. 120, no. 5, 721-749 (2010).

18. J.-F. Le Gall, Random trees and applications, Probability Surveys 2, 245-311 (2005).

19. J.-F. Le Gall, G. Miermont, Scaling limits of random planar maps with large faces, Ann. Probab, 39 (1), 1-69 (2011).

20. J.-F. Le Gall, Y. Le Jan, Branching processes in Lévy Processes: The exploration process, Ann. Probab., 26(1), 213-512 (1998).

21. J.-F. Marckert, A. Mokkadem, The depth first processes of Galton-Watson trees converge to the same Brownian excursion, Ann. Probab. 31, 1655-1678 (2003).

22. J. Pitman, Combinatorial Stochastic Processes, Lecture Notes Math. 1875. Springer-Verlag, Berlin (2006).

23. D. Revuz, M. Yor, Continuous martingales and Brownian motion, Third edition, Grundlehren der Mathematischen Wissenschaften [Fundamental Principles of Mathematical Sciences], 293. Springer-Verlag, Berlin (1999).

24. V.M. Zolotarev, One-Dimensional Stable Distributions, Vol. 65 of Translations of Mathematical Monographs , American Mathematical Society (1986).

Laboratoire de mathématiques,

UMR 8628 CNRS.

Université Paris-Sud

91405 ORSAY Cedex, France

igor.kortchemski@normalesup.org 